Research Article

\title{
PPAR-Gamma Agonist Pioglitazone Reduced CD68+ but Not CD163+ Macrophage Dermal Infiltration in Obese Psoriatic Patients
}

\author{
Ya. O. Yemchenko $\mathbb{D}$, V. I. Shynkevych $(\mathbb{D}$, K. Ye Ishcheikin, and I. P. Kaidashev \\ Ukrainian Medical Stomatological Academy, Poltava 36024, Ukraine \\ Correspondence should be addressed to Ya. O. Yemchenko; yanaumsa@ukr.net
}

Received 24 December 2019; Revised 24 February 2020; Accepted 6 April 2020; Published 1 May 2020

Academic Editor: Elisabetta Mueller

Copyright (c) 2020 Ya. O. Yemchenko et al. This is an open access article distributed under the Creative Commons Attribution License, which permits unrestricted use, distribution, and reproduction in any medium, provided the original work is properly cited.

\begin{abstract}
Background. Macrophages are of great importance in the development of obesity and psoriasis. Signaling via PPAR- $\gamma$ in certain macrophage populations is associated with M2-like features and anti-inflammatory profile. In this research, we evaluated the anti-inflammatory action of pioglitazone by the immunohistochemical study of M1 and M2 macrophages in psoriasis-affected skin in obese patients. Methods. We used immunohistochemistry to characterize CD68+ and CD163+ macrophages and pathomorphological description of skin biopsy, obtained from 6 obese psoriatic patients before and after treatment with 15, 30, and $45 \mathrm{mg}$ pioglitazone, once a day during 6 months. Two patients with conventional therapy and without pioglitazone served as control. Results. Generally, CD163+ cell quantities in psoriasis-affected skin significantly dominated over CD68+ before and after all treatment regiments. Among patients who received pioglitazone, some of them clearly responded to treatment from lowest to highest doses by decreasing CD68+ cells. In the group with $30 \mathrm{mg}$ pioglitazone regiment, we detected a significant reduction of CD68+ cells in dermal infiltrates: CI 95\% (16-32) before versus CI 95\% (2-7) after treatment. Pioglitazone dose escalation led to certain normalization of skin morphology. Conclusion. The immunohistochemical study allows us to show the anti-inflammatory effect of pioglitazone in psoriatic obese patients, which can be mediated by reducing the number of CD68+ macrophages, but not CD163+ macrophages, in the affected dermis.
\end{abstract}

\section{Introduction}

Psoriasis is a life-long, incurable, disfiguring, disabling, stigmatizing disease with a worldwide prevalence of about $2 \%$ [1]. It can manifest in many different forms and often accompanied by obesity [2].

The etiology and pathogenesis of psoriasis include many immunological mechanisms $[3,4]$, in which macrophages $(\mathrm{M} \varphi s)$ and their different populations are involved in the inflammatory cascade [5]. Comorbid obesity further complicates immunological disorders in psoriasis $[2,6]$.

The clinical studies showed the therapeutic effects of PPAR- $\gamma$-agonist pioglitazone (PGZ) in the treatment of cutaneous and metabolic pathologies in psoriasis $[7,8]$, but mechanisms of such effects remain unclear. PGZ is a specific agonist of PPAR- $\gamma$ from the thiazolidinedione group, in which PPAR- $\gamma$ stimulation led to a reduction in the expression of $\mathrm{NF} \kappa \mathrm{B}$ in mononuclear cells [9]. Macrophages PPAR- $\gamma$ can be one of the possible targets for PGZ [10].

In addition, $M \varphi s$ are of great importance in the development of obesity and psoriasis. Accumulated researches showed that pathogenesis of obesity and psoriasis has a common trait that causes a systemic inflammatory response. Excessive accumulation of adipose tissue, which produces adipokines and cytokines, is a source of chronic inflammation due to the involvement of macrophages capable of secreting and activating cytokines for inflammation. It is proved that fatty tissue has almost all known Toll-like receptors (TLRs). TLR ligands are both LPS of microorganisms and saturated fatty acids. Activation of fatty tissue TLRs leads to increased synthesis of adipokines, cytokines, and chemokines and stimulates the subsequent expression of TLRs 


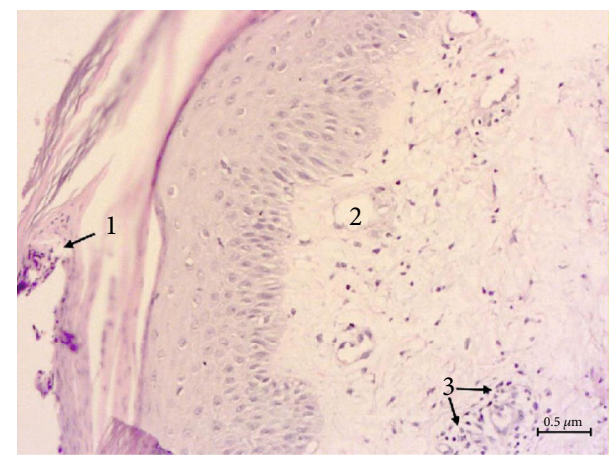

Figure 1: Pathomorphology of psoriasis-affected skin: Munro's microabscesses (1), dilated capillaries in the dermal papillae (2), and lymphohistiocytic infiltrate (3). Hematoxylin-eosin staining, $\mathrm{mg}, \times 200$. Scale bar, $0.5 \mu \mathrm{m}$.

[11]. Molecular mechanisms of chronic systemic inflammation are associated with proinflammatory nuclear transcription factor $\mathrm{NF} \kappa \mathrm{B}$ [12] and anti-inflammatory activity of PPARs, which directly modulate the activity of genes responsible for the condition and function of adipose tissue, lipid metabolism, the activity of inflammatory cells, and their production of cytokines, adhesion factors, cell differentiation, and apoptosis. PPAR-activated receptors are central between lipids and inflammation because lipids that stimulate chronic inflammation are PPAR-activating ligands also [13].

There are two main clinically significant subpopulations of $\mathrm{M} \varphi \mathrm{s}$ : classically (M1) and alternatively activated (M2) $[10,14] . \mathrm{M} 1$ and $\mathrm{M} 2 \mathrm{M} \varphi$ s have the ability to exhibit the polar-opposite $\mathrm{M} 2 /$ heal and M1/kill functions [15]. M $\varphi$ s polarization occurs against the background of significant metabolic changes [10]. In different diseases, the M1/M2 ratio can change, as does the expression profile of these cells, which has a pathogenic role $[16,17]$.

PPAR- $\gamma$ tickling of these subpopulations may lead to the development of anti-inflammatory effects [18-20].

CD68 and CD163 are used to identify $\mathrm{M} \varphi$ s in tissue sections [21]. CD163 is a hemoglobin-haptoglobin complexbinding scavenger receptor and one of several macrophage markers classified as markers of alternatively activated $\mathrm{M} \varphi$ s [22]. CD68 molecule is functionally important for M1 macrophages. As a receptor for oxidized low-density lipoproteins, it can activate M1 phagocytosis and promote proinflammatory cytokine production. CD68 is highly expressed on human monocytes and tissue $\mathrm{M} \varphi \mathrm{s}$ [21, 23]. The previous study demonstrated that macrophage marker CD68 was increased in psoriasis compared to normal skin, and coexpressed with CD163 [24].

Thus, in this research, we evaluated the anti-inflammatory action of pioglitazone by the immunohistochemical study of $\mathrm{M} 1$ and M2 M $\varphi$ s in psoriasis-affected skin.

\section{Materials and Methods}

2.1. Subject Recruitment. Prior to the study, the approval from the Academy's Bioethics Commission was obtained.

The study included 8 patients with the verified diagnosis of extensive psoriasis vulgaris, advanced stage, and moderate severity with concomitant obesity $30 \leq \mathrm{BMI}<40 \mathrm{~kg} / 1.73 \mathrm{~m}^{3}$ ( 3 women and 5 men, aged from 40 to 60 , the average age of 52 years). All patients signed a voluntary informed consent to participate in the study.

Patients were stratified and randomized $1: 1: 1: 1$; each group included 2 patients. The 1 st group of patients received standardized conventional therapy-sedation, detoxifying agents, antihistamine, hepatoprotectors, vitamins, and $1-2 \%$ salicylic ointment 2 times a day topically. Patients of groups 2,3 , and 4 were prescribed pioglitazone at a dose of 15, 30, and $45 \mathrm{mg}$, respectively, orally once a day for 28 days, additionally to the treatment protocol.

2.2. Punch Biopsy. We conducted an in-depth descriptive study of macrophage subpopulations on skin biopsies. The samples were obtained by punch biopsy of psoriatic plaques from the skin areas of lower extremities exactly before treatment and on the 28th day. Punch biopsy was performed using a special tubular scalpel (Dermo-punch) with a diameter of $3 \mathrm{~mm}$, under local infiltration anesthesia with lidocaine hydrochloride $2 \%$. The biopsies were fixed in a $10 \%$ solution of neutral buffered formalin and embedded in paraffin.

2.3. Immunohistochemical Studies. We performed immunohistochemical studies using the streptavidin peroxidase method. Paraffin sections, $3 \mu \mathrm{m}$ thick, were deparaffinized and dehydrated, antigens were recovered in citrate buffer in the microwave oven, and endogenous peroxidase was blocked. Further, the sections were incubated at $4^{\circ} \mathrm{C}$ overnight with murine monoclonal antibodies anti-CD68 (1:30, clone PGM1, REF PD M065-S, Diagnostic BioSystems, USA) and anti-CD163 (1:100, clone 10D6, REF Mob460-01, Diagnostic BioSystems, USA). Afterwards, the sections were treated in two steps with the Mouse/Rabbit PolyVue ${ }^{\mathrm{TM}} \mathrm{HRP} / \mathrm{DAB}$ Detection System (Diagnostic BioSystems, USA), with visualization by chromogen; the nuclei were counterstained with Mayer's haemalaun and enclosed under the cover glass. We used Antibody Diluent buffer as a negative control instead of primary antibodies, and lymph node tissues were used as a positive control.

Quantitative indicators were obtained by counting immunopositive CD68+ and CD163+ cells over the entire field of view with a large magnification lens $\times 40$ (high power field, HPF) of the dermal region. We took into account all obtained quantitative individual data from all fields of view without calculating the mean. Microphotographs were obtained using the ZEISS Axio Lab.A1 microscope, Carl Zeiss MicroImaging GmbH (mg; ×200, ×400).

2.4. Statistical Analysis. We conducted that the statistical analysis of comparisons between groups was performed using the GraphPad Prism 5 software with parametric $T$ test (if normal distribution) and nonparametric methods: Wilcoxon rank data analysis for dependent variables and Spearman correlation.

\section{Results}

The pathomorphological pattern of the psoriasis-affected specimens in all patients had characteristic signs of this 


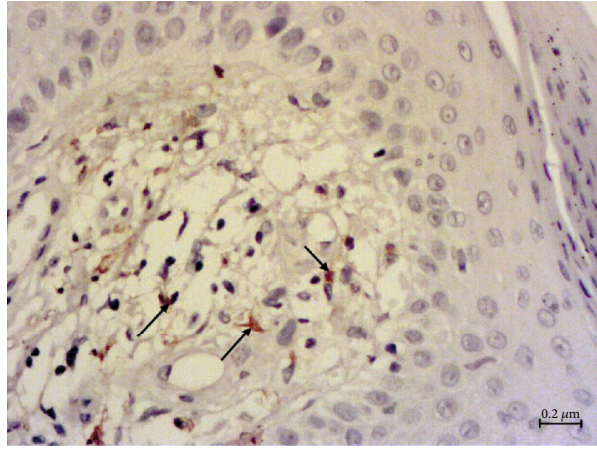

(a)

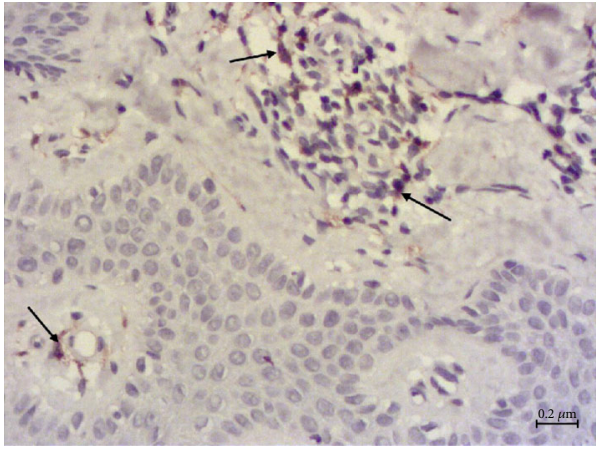

(b)
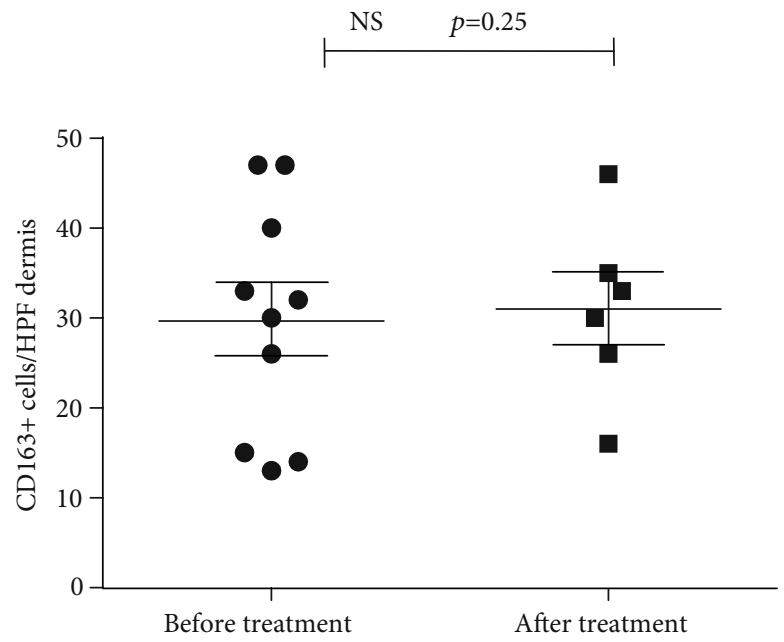

(c)

Figure 2: Immunohistochemical detection of CD163+ cells in the psoriatic plaque biopsy specimen before (a) and after treatment with 30 mg pioglitazone (b). CD163+ cells (arrows) are rather tightly represented in the dermal papillae surrounding the vessels. Contrasting: Mayer's haemalaun, $\mathrm{mg}, \times 400$. Scale bars, $0.2 \mu \mathrm{m}$. CD163+ cell number in all groups did not differ significantly before and after treatment (c).

disease. We detected the typical changes of the epidermis, such as accumulation of neutrophils in the stratum corneum of the epidermis: Munro's microabscesses, decreased thickness of the epidermis as compared to the length of dermal papillae, regular acanthosis, often with dentate ridges of the epidermis, and dilated capillaries in the papillae. Lymphohistiocytic infiltrates of various densities were localized subepidermally in the dermal papillae (Figure 1).

In psoriatic skin lesions, general characteristics of CD68+ and $\mathrm{CD} 163+$ were as follows: immunoreactivity was observed in the form of brown granular staining of cells, which revealed their different size and shape features; CD68 + and CD163+ cells in psoriatic skin were localized in the dermal papillae and deeper dermis, along and around the dilated superficial vessels, as well as in the composition of lymphohistiocytic infiltrates. We found the largest number of immunopositive cells in the papillary and reticular layers of the dermis (Figures 2-4).

Visually, the number of CD163+ cells dominated in all samples. The assessment of CD68+/CD163+ ratio, obtained by the $T$-test for dependent variables, confirmed a significantly higher fraction of CD163+ cells $(p=0.0002)$, with no significant correlation between CD68+ and CD163+ quanti- ties. The average number of CD68+ was 10 cells (per HPF of papillary and reticular layers of dermis), confidential interval (CI) $95 \%$ (6-14), whereas the number of CD163+ was 22 cells, CI 95\% (17-26).

After treatment, the ratio did not change, and CD163+ $\mathrm{M} \varphi s$ prevailed over CD68+ $(p=0.0002$, Wilcoxon method $)$ in all groups.

Numerous CD163+ cells in psoriasis were observed in at least two forms: small-sized cells with a small nucleus, as in lymphocytes, and relatively large branched cells; these varieties persisted after treatment. Both types of cells were localized in essentially the same areas and had different shapes depending on the position. Hence, the slightly larger ones were on the tips of the papillae, whereas smaller cells were in the composition of lymphohistiocytic infiltrates, and/or flattened spindle-shaped-in the reticular layer. The typical localization was along the vessels of the dermal papillae, which were observed specifically for CD163+ cells, where they formed a kind of the first line (Figure 2), as compared to $\mathrm{CD} 68+$ (Figure 3). CD68+ M $\varphi$ s also occurred in different shapes and sizes, slightly sparse in infiltrates, and presented in the dermal papillae, sometimes not in all, around the capillaries (Figure 4). 


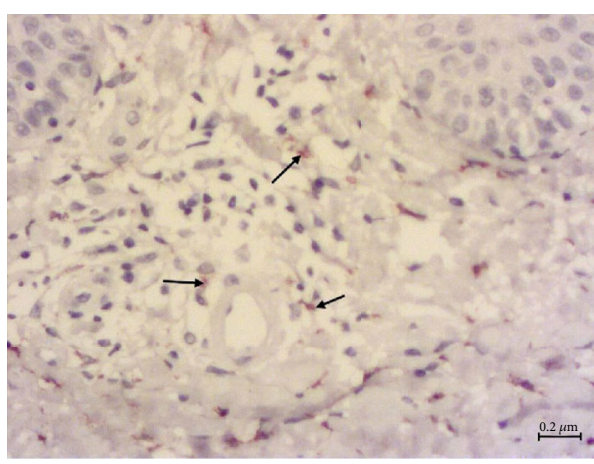

(a)

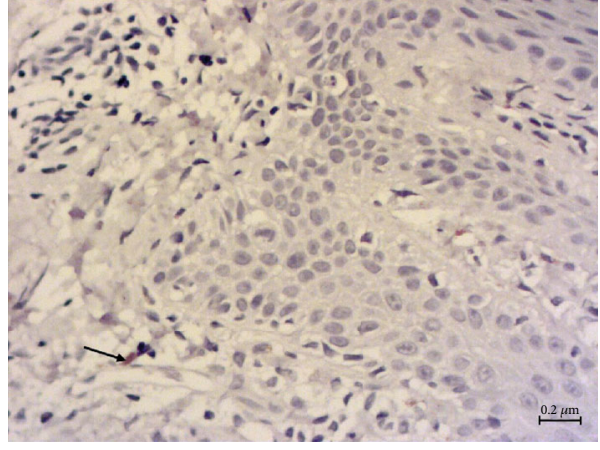

(b)

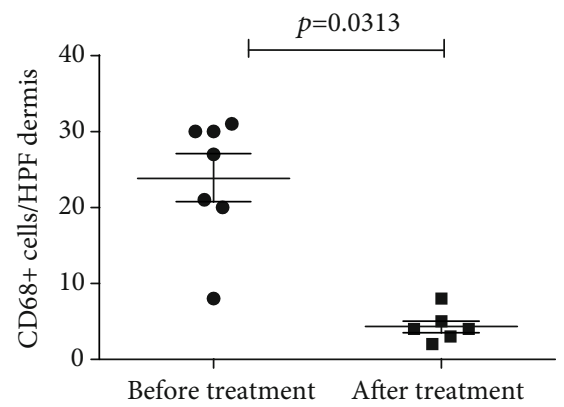

(c)

Figure 3: Immunohistochemical detection of CD68+ cells in psoriatic plaque biopsy specimens. (a) CD68+ cells (arrows) in the infiltrate and the reticular layer of the dermis before treatment. (b) Single CD68+ cells (arrow) after the treatment with 30 mg pioglitazone. Contrasting: Mayer's haemalaun, $\mathrm{mg}, \times 400$. Scale bars, $0.2 \mu \mathrm{m}$. (c) CD68+ cells reduced significantly (Wilcoxon method).

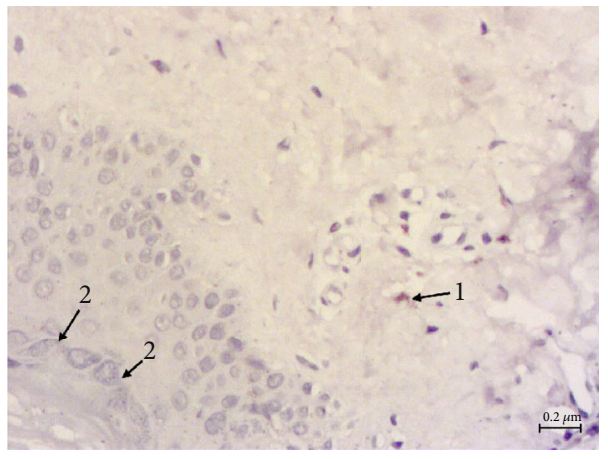

(a)

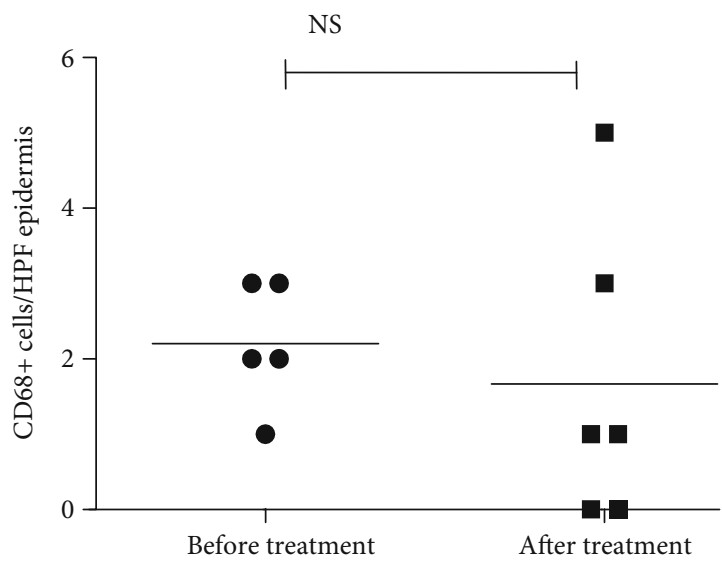

(b)

FIGURE 4: Immunohistochemical detection of CD68+ cells in skin biopsy specimens after treatment of psoriasis with pioglitazone $45 \mathrm{mg}$. (a) Single immunopositive cell (1) and granular layer (2). Contrasting: Mayer's haemalaun, mg, $\times 400$. Scale bar, $0.2 \mu \mathrm{m}$. (b) CD68+ cell number in the patient did not differ significantly.

The morphological and quantitative comparison of $\mathrm{CD} 163+$ cells before and after treatment demonstrated no significant differences in each group and in total (Figure 2).

In the group with conventional therapy, comparison of CD68+ cells before and after treatment demonstrated no statistically significant changes or morphologic differences $(p=$ 0.343 , data not shown).

A personalized approach justifies close attention to each patient. Therefore, the study revealed the patient who clearly responded to the treatment with the $15 \mathrm{mg}$ of PGZ, in whom CD68+ cells almost completely disappeared after treatment.

In the group with $30 \mathrm{mg}$ PGZ, the statistical comparison of CD68+ cells (Wilcoxon method) showed a significant decrease. Treatment with $30 \mathrm{mg}$ PGZ use decreased the number of CD68+ cells from CI 95\% (16-32) to CI 95\% (2-7) significantly (Figure 3 ).

Besides, in one patient after the treatment with $45 \mathrm{mg}$ of PGZ, dermal papillae presented on the preparation were 
smoothed, epidermal ridges were rounded, and orthokeratosis (morphological signs of some normalization of the skin condition) and only single CD68+ cells were observed, which can be interpreted as a decrease in the infiltration density (Figure 4).

\section{Discussion}

The involvement of $\mathrm{M} \varphi \mathrm{s}$ in the pathogenesis of psoriasis is a long-standing but actual research issue. In psoriasis, $\mathrm{M} \varphi s$ are involved in the perception of danger signals. Their activation by the proinflammatory cytokines IL- 6 and TNF- $\alpha$ and chemokines CXCL8, CCL5, CXCL1, and CXCL2 involves other inflammatory cells [25]. It is known that the number of $\mathrm{M} \varphi \mathrm{s}$ increases significantly in psoriasis-affected tissues [26]. The M1/M2-type macrophages drove $\mathrm{T}$ cell differentiation to Th1- or Th2-like phenotype, respectively. Such macrophageinnate activities are the central directing element in immune responses which is a drastic change in understanding how immune systems operate. Most importantly, this revelation is opening up whole new approaches to immunotherapy [27].

The data on $\mathrm{M} \varphi s$ polarization under the influence of PPAR- $\gamma$ agonists and antagonists are steadily growing, mainly in animal models [18-20]. Signaling via PPAR- $\gamma$ in the individual $\mathrm{M} \varphi$ s populations is associated with $\mathrm{M} 2$-like features, including the anti-inflammatory ones [28].

Thus, we investigated the influence of PPAR- $\gamma$ agonist PGZ on M1 and M2 M $\varphi$ s of psoriasis-affected skin, by immunohistochemical study. Our data showed a decrease in the CD68+ $M \varphi$ s population by different doses of PGZ. CD68 marker was used in a recent study as one of the identifiers for inflammatory M1 [29]. The reduction of CD68+ $\mathrm{M} \varphi s$ has correlated with clinical success in psoriatic patients. The numbers of phosphorylated $\mathrm{I} \kappa \mathrm{B} \alpha$ activated $\mathrm{CD} 68+\mathrm{M} \varphi$ s were reduced in psoriasis plaques after treatment with new biomaterials based on nanoparticles significantly [29]. Therefore, a decrease of CD68+ M1 can explain the success of PGZ treatment.

In obese psoriatic patients, CD163+ cells were the predominant population in the affected skin, compared to CD68+. Conventional treatment did not influence this ratio. The prevalence of $\mathrm{CD} 163+$ cells as alternative activated $\mathrm{M} \varphi s$ goes in parallel with angiogenesis de novo in psoriatic skin. The descriptive morphological comparison of CD163+ cells before and after the treatment with PGZ showed no drastic changes.

Our data support the idea that $\mathrm{CD} 163+$ cells drive immune-mediated proliferation in these patients [30]. On the other hand, the controversial data about CD163+ cell classical activation in psoriasis were published [24].

PPAR- $\gamma$-dependent activation of macrophages is obviously capable of restoring the vital cleaning or clearance functions of macrophages in some infections, preventing excessive inflammation in septic conditions [28]. Therefore, the administration of PGZ may also influence CD163+ M2 $\mathrm{M} \varphi s$, although their quantitative and morphological changes could not be recorded in this study.

Additional treatment with PGZ reduced the number of CD68+ cells starting at a dose of $15 \mathrm{mg}$; statistical significance was registered at a dose of $30 \mathrm{mg}$. Furthermore, in the dose of $45 \mathrm{mg}$, there were also signs of the decreased density of CD68+, together with morphological manifestations of regression of psoriatic plaques: thickening of the epidermis, orthokeratosis, reduced height of the dermal papillae, and lack of branching of the epidermal ridges. Thus, PGZ dose escalation led to certain normalization of skin morphology.

CD68 is a type I integral membrane protein with a heavily glycosylated extracellular domain and binds to tissueand organ-specific lectins or selectins. The protein is a member of the scavenger receptor family, with typical functions which are cellular debris removal, phagocytosis promotion, and $\mathrm{M} \varphi$ s recruitment. Thus, CD68 contributes to inflammation support [31].

Taken together with clinical indicators in the dynamics of psoriasis treatment with PGZ, such as a decrease of PASI index and reduction of relapse rate up to 1-2 times per year $[7,8,32]$, decreasing of CD68+ cells indicates inhibition of inflammation locally.

The limitations of the study were a small number of patients due to ethical reasons and using of $\mathrm{M} \varphi s$ markers that can partially overlap in pathology $[16,17,24,29]$.

Further research will be targeted on the investigation of M1/M2 cytokine levels under PGZ influence on obese psoriatic patients.

\section{Conclusions}

The immunohistochemical study allows us to show the antiinflammatory effect of pioglitazone in psoriatic obese patients, which can be mediated by reducing the number of CD68+ macrophages, but not CD163+ macrophages, in the affected dermis.

\section{Data Availability}

The data used to support the findings of this study are available from the corresponding author upon request.

\section{Conflicts of Interest}

The authors declare that there are no conflicts of interest regarding the publication of this paper.

\section{Acknowledgments}

The study was a part of the research No. 0120 U101166 "The study of the pathogenetic role of the circadian molecular clock in the development of metabolic diseases and systemic inflammation and the development of treatment methods aimed at these processes" funded by Ukrainian Ministry of Health Public Service.

\section{References}

[1] World Health Organization (WHO), "Global report on psoriasis," Geneva, Switzerland; 2016.

[2] A. Egeberg, P. Gisondi, J. M. Carrascosa, R. B. Warren, and U. Mrowietz, "The role of the interleukin-23/Th17 pathway in cardiometabolic comorbidity associated with psoriasis," 
Journal of the European Academy of Dermatology and Venereology, 2020.

[3] J. E. Hawkes, B. Y. Yan, T. C. Chan, and J. G. Krueger, "Discovery of the IL-23/IL-17 signaling pathway and the treatment of psoriasis," The Journal of Immunology, vol. 201, no. 6, pp. 1605-1613, 2018.

[4] Y. O. Yemchenko, K. E. Ishcheykin, and I. P. Kaidashev, "Modern views of the immunopathogenesis of psoriasis disease," World of Medicine and Biology, vol. 14, no. 65, article 2079-8334-2018-3-65-134-139, pp. 134-139, 2018.

[5] J. G. Krueger and P. M. Brunner, "Interleukin-17 alters the biology of many cell types involved in the genesis of psoriasis, systemic inflammation and associated comorbidities," Experimental Dermatology, vol. 27, no. 2, pp. 115-123, 2018.

[6] W.-H. Boehncke, "Systemic Inflammation and Cardiovascular Comorbidity in Psoriasis Patients: Causes and Consequences," Frontiers in Immunology, vol. 9, 2018.

[7] G. Chang, J. Wang, J. Song, Z. Zhang, and L. Zhang, "Efficacy and safety of pioglitazone for treatment of plaque psoriasis: a systematic review and meta-analysis of randomized controlled trials," Journal of Dermatological Treatment, pp. 1-7, 2019.

[8] N. Shafiq, S. Malhotra, P. Pandhi, M. Gupta, B. Kumar, and K. Sandhu, "Pilot trial: pioglitazone versus placebo in patients with plaque psoriasis (the P6)," International Journal of Dermatology, vol. 44, no. 4, pp. 328-333, 2005.

[9] N. L. Kutsenko, L. E. Vesnina, and I. P. Kaĭdashev, "Pioglitazone, an activator of PPAR-gamma, reduces the expression of $\mathrm{kB}$ nuclear factor and inhibits apoptosis in mononuclear cells of peripheral blood in vitro," Fiziologicheskiu Zhurnal, vol. 58, no. 2, pp. 33-38, 2012.

[10] I. P. Kaidashev, "Polarization of macrophages and regulation of the immune response (literature review and the author's own research)," Journal of the National Academy of Medical Sciences of Ukraine, vol. 239, no. 1-2, pp. 9-22, 2017.

[11] Z. Liu, T. Xiao, X. Peng, G. Li, and F. Hu, “APPLs: more than just adiponectin receptor binding proteins," Cellular Signalling, vol. 32, pp. 76-84, 2017.

[12] Higher State Educational Establishment of Ukraine "Ukrainian Medical Stomatological Academy", Poltava, Ukraine and I. P. Kaĭdashev, "NF-kB activation as a molecular basis of pathological process by metabolic syndrome," Fiziologicheskil Zhurnal, vol. 58, no. 1, pp. 93-101, 2012.

[13] O. V. Byelan, T. Mamontova, L. Vesnina, O. A. Borzykh, and I. Kaidashev, "Anti-inflammatory and endothelium protective effect of long-term pioglitazone intake in patients suffering from bronchial asthma concurrent with ischemic heart disease," Wiadomosci lekarskie, vol. 70, no. 4, pp. 712-720, 2017.

[14] C. D. Mills, A. C. Thomas, L. L. Lenz, and M. Munder, "Macrophage: SHIP of immunity," Frontiers in Immunology, vol. 5, 2014.

[15] C. D. Mills, L. L. Lenz, and K. Ley, "Macrophages at the fork in the road to health or disease," Frontiers in Immunology, vol. 6, 2015.

[16] T. Satoh, "Functional diversity of disorder-specific macrophages," Rinsho Ketsueki, vol. 59, no. 6, pp. 805-811, 2018.

[17] S. Gordon, A. Plüddemann, and F. M. Estrada, "Macrophage heterogeneity in tissues: phenotypic diversity and functions," Immunological Reviews, vol. 262, no. 1, pp. 36-55, 2014.

[18] J. Ji, T. F. Xue, X. D. Guo et al., “Antagonizing peroxisome proliferator-activated receptor $\gamma$ facilitates M1-to-M2 shift of microglia by enhancing autophagy via the LKB1-AMPK signaling pathway," Aging Cell, vol. 17, no. 4, article e12774, 2018.

[19] W. Luo, Q. Xu, Q. Wang, H. Wu, and J. Hua, "Effect of modulation of PPAR- $\gamma$ activity on Kupffer cells M1/M2 polarization in the development of non-alcoholic fatty liver disease," Scientific Reports, vol. 7, no. 1, 2017.

[20] Z. Chen, P. Yuan, X. Sun et al., "Pioglitazone decreased renal calcium oxalate crystal formation by suppressing M1 macrophage polarization via the PPAR- $\gamma$-miR-23 axis," American Journal of Physiology-Renal Physiology, vol. 317, no. 1, pp. F137-F151, 2019.

[21] M. H. M. Barros, F. Hauck, J. H. Dreyer, B. Kempkes, and G. Niedobitek, "Macrophage polarisation: an immunohistochemical approach for identifying M1 and M2 macrophages," PLoS One, vol. 8, no. 11, article e80908, 2013.

[22] B. O. Fabriek, C. D. Dijkstra, and T. K. van den Berg, "The macrophage scavenger receptor CD163," Immunobiology, vol. 210, no. 2-4, pp. 153-160, 2005.

[23] S. Tedesco, C. Bolego, A. Toniolo et al., "Phenotypic activation and pharmacological outcomes of spontaneously differentiated human monocyte-derived macrophages," Immunobiology, vol. 220, no. 5, pp. 545-554, 2015.

[24] J. Fuentes-Duculan, M. Suárez-Fariñas, L. C. Zaba et al., "A subpopulation of CD163-positive macrophages is classically activated in psoriasis," Journal of Investigative Dermatology, vol. 130, no. 10, pp. 2412-2422, 2010.

[25] G. Arango Duque and A. Descoteaux, "Macrophage Cytokines: Involvement in Immunity and Infectious Diseases," Frontiers in Immunology, vol. 5, 2014.

[26] Y. Wang, R. Edelmayer, J. Wetter et al., "Monocytes/Macrophages play a pathogenic role in IL-23 mediated psoriasis- like skin inflammation," Scientific Reports, vol. 9, no. 1, article 41655, p. 5310, 2019.

[27] C. D. Mills and K. Ley, "M1 and M2 macrophages: the chicken and the egg of immunity," Journal of Innate Immunity, vol. 6, no. 6, pp. 716-726, 2014.

[28] C. M. Leopold Wager, E. Arnett, and L. S. Schlesinger, "Macrophage nuclear receptors: emerging key players in infectious diseases," PLoS Pathog, vol. 15, no. 3, article e1007585, 2019.

[29] D. Crisan, K. Scharffetter-Kochanek, M. Crisan et al., "Topical silver and gold nanoparticles complexed withCornus massuppress inflammation in human psoriasis plaques by inhibiting NF- $\kappa$ B activity," Experimental Dermatology, vol. 27, no. 10, pp. 1166-1169, 2018.

[30] T. Akhtar, W. Y. Wani, M. A. Kamal, and R. Kaur, "Role of angiogenic growth factors in psoriasis: a review," Current Drug Metabolism, vol. 19, no. 11, pp. 910-916, 2018.

[31] D. Chistiakov, M. Killingsworth, V. Myasoedova, A. N. Orekhov, and Y. V. Bobryshev, "CD68/macrosialin: not just a histochemical marker," Laboratory Investigation, vol. 97, no. 1, pp. 4-13, 2017.

[32] R. M. El-Gharabawy, A. S. Ahmed, and A. H. Al-Najjar, "Mechanism of action and effect of immune-modulating agents in the treatment of psoriasis," Biomedicine \& Pharmacotherapy, vol. 85, pp. 141-147, 2017. 